Document downloaded from:

http://hdl.handle.net/10251/82441

This paper must be cited as:

Jimenez, JM.; Lloret, J.; Díaz Santos, JR.; Lacuesta Gilaberte, R. (2017). Test bench to test protocols and algorithms for multimedia delivery. En Lecture Notes of the Institute for Computer Sciences, Social Informatics and Telecommunications Engineering. Springer Verlag. 124-134. doi:10.1007/978-3-319-49580-4_12.

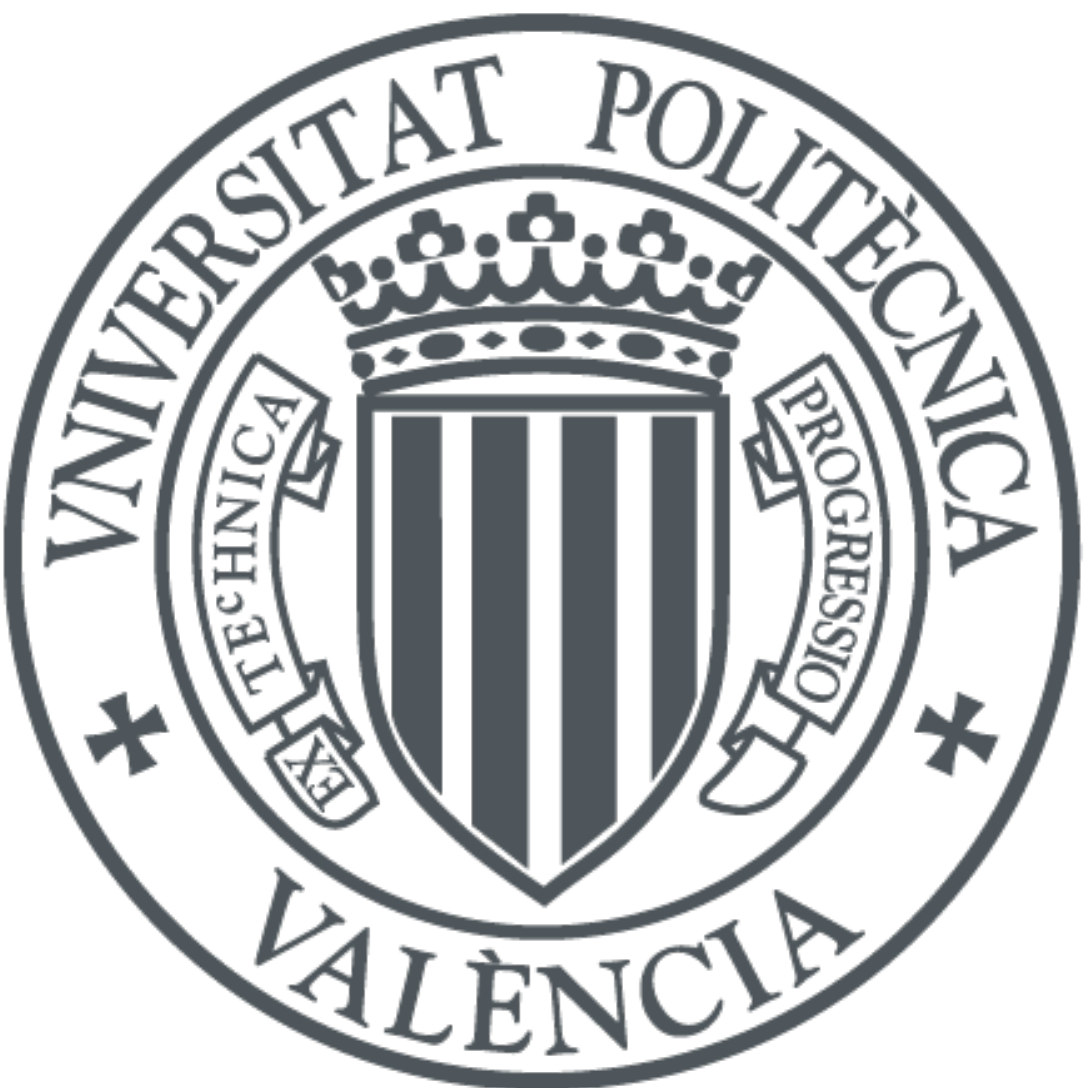

The final publication is available at

https://link.springer.com/chapter/10.1007/978-3-319-49580-4_12

Copyright Springer Verlag

Additional Information 


\title{
Test Bench to Test Protocols and Algorithms for Multimedia Delivery
}

\author{
Jose M. Jimenez ${ }^{1}$, Jaime Lloret ${ }^{1}$, Juan R. Diaz ${ }^{1}$, Raquel Lacuesta ${ }^{2}$ \\ ${ }^{1}$ Instituto de Investigación para la Gestión Integrada de Zonas Costeras \\ Universidad Politécnica de Valencia, Camino Vera s/n, 46022, Valencia, Spain \\ ${ }^{2}$ Escuela Politécnica Superior de Teruel \\ Universidad de Zaragoza, C/ Ciudad Escolar s/n, 44003, Teruel, Spain \\ \{jojiher,jlloret\}@dcom.upv.es, juanramon.diazsantos@gmail.com, lacuesta@unizar.es
}

\begin{abstract}
Nowadays, the study of Quality of Service (QoS) in multimedia streaming is generally carried out by simulators. However, the results of these tests are not quite real. This forces researchers to work with approximations. In this paper, we implement a test bench to test the performance and assess the protocols and algorithms for multimedia delivery. This test bench let us evaluate the network parameters performance, by manipulating the devices under controlled conditions, and allow us to identify different application cases. We can confirm that the designed test bench gives us more real measures than a simulators, which allows us to do many types of tests with low cost.
\end{abstract}

Keywords: Multimedia, Test bench, QoS, Protocols and Algorithms.

\section{Introduction}

The evolution of multimedia delivery has been possible thanks to the development of control mechanisms to promote Quality of Service (QoS) and Quality of Experience (QoE) in IP networks: traffic prioritization, streaming protocols, etc. [1][2]. They have brought benefits to both service operators and users in many aspects such as: obtaining an efficient communication, optimizing resources and reducing costs in both, the part of the service providers and in consequence to the users, optimizing network administration, delivery of new services, and efficient and secure network infrastructure developing. New technologies such as adaptive streaming [3] and Software Defined Networks [4] are providing research advances in this topic.

A QoS categorized model of user-centric let us know the performance quality goals of audio, data and video. These goals are detailed in the Recommendation UIT- T G.1010 [5] and Recommendation UIT- R M.1079-2 [6]. So, we primarily must take into account parameters such as latency, jitter, and bandwidth and packet loss in both wired and wireless networks [7][8]. Others parameters we are interested in are shown in Cisco VNI report [9]. Some of them are internet user demand, network connections growth, as well as the generation of internet video minutes per month in a global scale and especially the number of video users in Internet.

In this work, we study the effect of stressing the network. In order to achieve this goal, we study the most well-known network parameters such as latency, jitter and 
number of lost packets, which provide relevant information to the network operators. We test different cases and analyze the received video quality. It is performed for both wireless and wired networks. First, we get a baseline that is later used to compare different study cases. These cases are designed taking into account the impact of multimedia delivery when there are few network resources.

The paper is organized as follows. In next section, we show several related works that include testbeds to show the performance of the network when there is multimedia delivery. In section 3, we describe the test bench implemented to test the case studies and the experiments. Section 4 explains the case studies as well as the experimentation process. In Section 5 we analyze the results. Finally, in Section 6, we show the conclusions we have reached and our future work.

\section{Relate Work}

This section shows the most relevant related works.

In [10], Lu et al. show the potential benefits of a "quality-based" adaptation approach for video applications. They discuss the joint impact of packet loss and encoding rate on video quality, based on which a simple quality feedback-control system has been built. Adaptation is carried out by measuring the quality of the received video and comparing it to a baseline reference. Their preliminary experimental results show both the viability and the benefits of using video quality as the basis of adaptation. Lee et al [11] introduced an IP Multimedia Subsystem (IMS) based testbed which provides a platform for the study of real-time services integration and orchestration. This open-source based testbed is built on the principle of Service Oriented Architecture (SOA), with an emphasis for real-time network services. They developed service-oriented system functionalities such as optical network connection and bandwidth management, mobile client authentication protocols, and video streaming services. They are packaged as interoperable service components, so that they can be integrated and orchestrated through their respective standard interfaces. Finally, they elaborated a proof-of-concept environment via a use-case scenario of having various client-server interactions over a heterogeneous network environment.

The work presented by $\mathrm{Mu}$ et al. in [12] introduces a system where digital video can be corrupted according to established loss patterns and the effect is measured automatically. The corrupted video is then used as input for user tests. Their results are analyzed and compared with the automatically generated. Within this paper they present the complete testing system that makes use of existing software as well as introducing new modules and extensions. The system can test packet loss in H.264 coded video streams and produce a statistical analysis detailing the results. The user tests can also be used to test the effectiveness of the objective video quality assessment models. Thus, the testbed is designed to provide a common testing platform on which objective and subjective evaluations can be performed with different testing scenarios (such as codec, transmission pattern) with the least human intervention (in terms of set-up and analysis). In [13], Kuwadekar et al present the performance results obtained on a IP Multimedia Subsystem (IMS) based Next Generation Network test-setup connected to a live operator network. These results are 
based on the Quality of Experience (QoE) that end user perceive. They can help service operators to deliver content rich applications to the end users as per their expectations. The aim of this work was to understand, with a series of experiment, the behavior of video streaming across various devices and changing network conditions. Through passive measurements, they characterized the behavior of video streaming and evaluated the users QoE.

\section{Description of the test bench design}

In order to perform the test bench design, we have taken into account the current technologies used by service providers and the usual features of the networks focused on video streaming. The equipment include a Cisco 2811 Router, a Cisco Catalyst 3560 Switch WS-C3560-24PS-E, a Cisco AIR-AP 1231G-E-K9 Access Point, 3 PC's with Intel Core Processor i5-2400, CPU @3.10 Ghz, 4Gb Memory RAM, Wireless adapter LINKSYS WUSB600N and Ethernet Intel $82579 \mathrm{~V}$ gigabit network connection and Win7 Operative System, and 1 PC with Intel Core Processor i5-2400 CPU @3.10 Ghz, 4Gb Memory RAM, Wireless adapter Cisco AIR-PI21AG-E-K9, $802.11 \mathrm{a} / \mathrm{b} / \mathrm{g}$, Ethernet Intel $82579 \mathrm{~V}$ gigabit network connection; and Windows XP Professional Service Pack 3 Operative System. The video used to perform the test was the Big Buck Bunny, Sunflower version, which is accessible at [14]. We use FFmpeg program [15] to encode the video with the following features: video time 35 seconds, encoded in H.264 [16], with a resolution of $1024 \times 768$ at $30 \mathrm{fps}$ (frames per second) and a bitrate of $1338 \mathrm{Kbps}$. We use VLC media player, version 2.1.3, for both server and client. To capture and analyze network parameters, under the Real Time Protocol (RTP), we use Wireshark, version 1.10.5. The main analyzed parameters are the delay (interval between two consecutive packets), jitter (deviation of each packet with respect to the latency), packet loss and BW (bandwidth).

\subsection{Physical topology}

The proposed topology consists of different LANs connected by serial interfaces. The LANs use IEEE 802.3 and IEEE 802.11 technology, while the serial connections use ISO 3309 and ISO 4335 (HDLC) technology. We used Routers Cisco 2811 to connect the LANs. In the LANs we used switches Cisco Catalyst 3560-24PS, which support IEEE 802.1q protocol and Cisco PVST + (Plus per vlan spanning tree). We configured the switch interfaces in different VLANs for each end user. The proposed physical topology can be observed in figure 1 .

\subsection{Logical topology}

The logical topology used VLANs, which allow building networks even when they are not sharing the same physical medium. The proposed logical topology can be observed in figure 2 . 

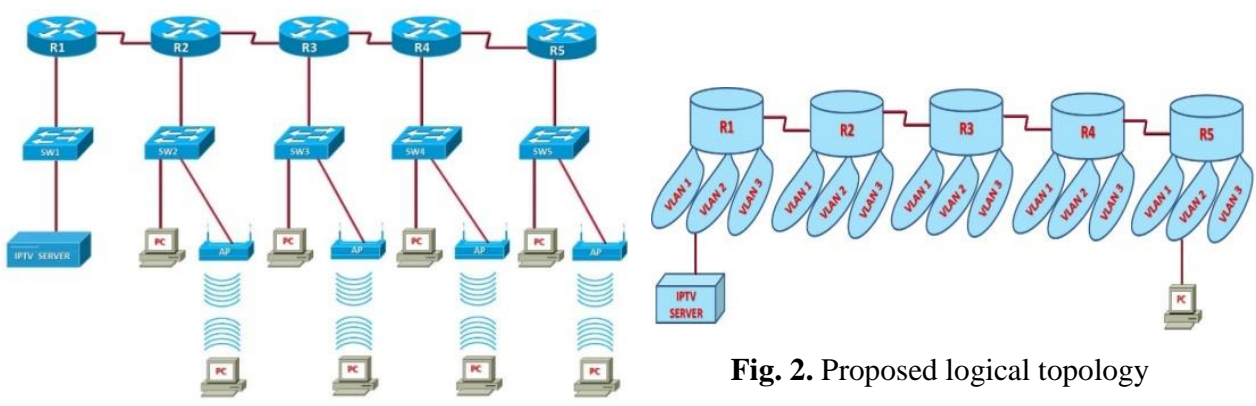

Fig. 1. Proposed physical topology

Fig. 2. Proposed logical topology

\section{Cases of study and experimentation}

In this section we develop a testbed for media streaming, which allow us to perform a series of actions based on network parameters, both internal and external, in order to evaluate the influence on the quality of the received video. The study is divided as follows: first we extract the base line on video streams both wired and wireless, then, we study the effect on the external and external parameters in such streaming.

\subsection{Base Line Study in Wired / Wireless Video Delivery}

For the study of the base line we used an IPTV server located at five hops between the VLC Clients (one in the wireless and another in the wired LAN), as shown in figure 3. This study allows evaluating the quality of video in the reception when there is no internal or external factor affecting the transmission delivery. At the reception, we use Wireshark to capture and evaluate the network parameters.

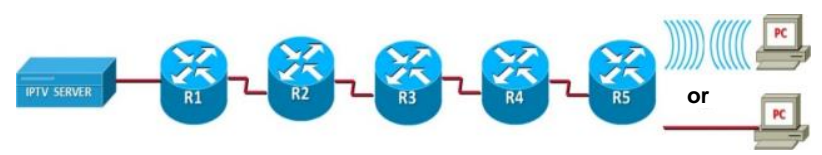

Fig. 3. Transmission from an IPTV server to a VLC Client (wireless or wired).

We conduct a series of experiments using this network design to gather measurements of network parameters. They will be the basis for analyzing and comparing them with respect to other study case. For the extraction of these measures we have delivered video through the network and we used Wireshark to capture some network parameters such as jitter, delay, packet loss and bandwidth. These parameters will serve as reference for the analysis of the study case. The results obtained for the delay, in both wired and wireless, are shown in the figures 4 and 5 . As can it be seen, the delay at the customer side, in both wired and wireless connection, had similar results. It is detected an almost constant delay of approximately $18 \mathrm{~ms}$ along the time. Furthermore the highest peak has $130 \mathrm{~ms}$, which is acceptable as it does not exceed the period recommended by the ITU. 


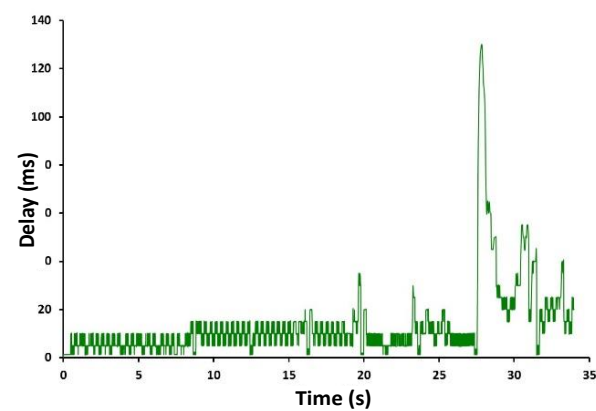

Fig. 4. Delay of the wired baseline.

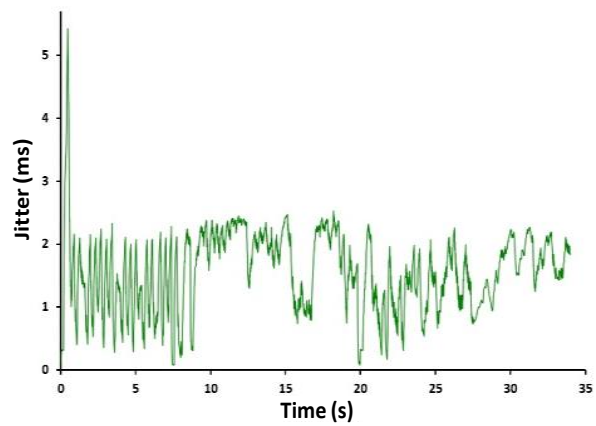

Fig .6. Jitter of the wired base line.

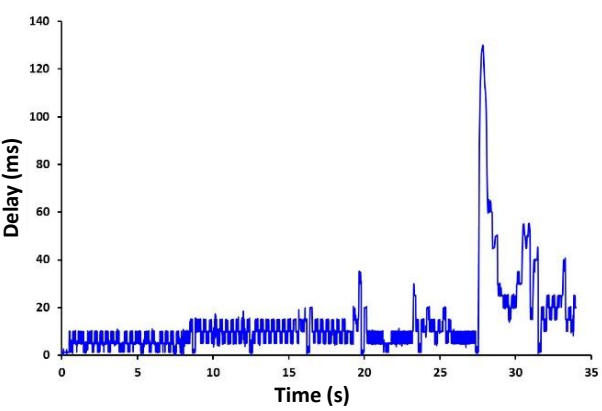

Fig. 5. Delay of the wireless baseline.

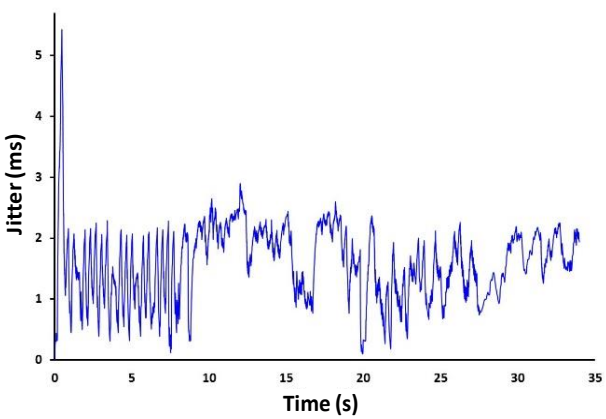

Fig. 7. Jitter of the wireless base line.

Figures 6 and 7 show the jitter at VLC clients side. The results show a peak of 5.5 $\mathrm{ms}$ at the beginning of the reception of the video until it stabilizes. Then, the graph follows an almost constant pattern that reaches a value of approximately of $2.5 \mathrm{~ms}$.

To estimate the bandwidth over the time, we calculated at each instant the average bandwidth consumed by the last 50 packets received. Considering that the average size of the transmitted packet is 1370 bytes, the bandwidth $\mathrm{BW}_{t}$ at time $T_{t}$ in which is received the 'i' packet is calculated by the equation (1).

$$
B W_{i}=\frac{N^{\circ} \text { bits }}{\text { Time interval }}=\frac{1370 * 50 * 100000}{\left(T_{i}-T_{i-50}\right) * 1000}
$$

Where, $\mathrm{T}_{\mathrm{i}-50}$ indicates the time instant reception of packet number 'i-50'. Factors 100000 and 1000 have been included to normalize the time, which originally are given in tens of microseconds, and bits units respectively, so that the result is displayed in Kbps. Figures 8 and 9 show the estimated bandwidth timely manner at each instant of time. It is possible to observe that in both cases, the mean values consumed at the reception, for both wired and wireless network, range approximately 2 Mbps. It also exists values that can exceed 9 Mbps. Finally, remark with respect to the loss of packets, that all transmitted packets (5092 packets), have been successfully received. This data will also be included in the baseline. Since neither losses of packages nor mistakes exist in the transmission, we obtained an optimal quality of video reception. 


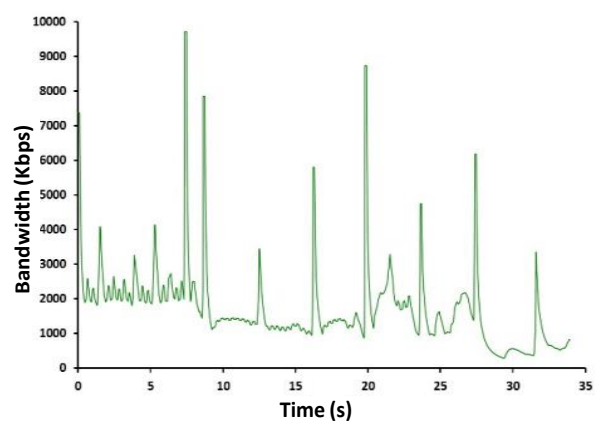

Fig. 8. Bandwidth wired base line.

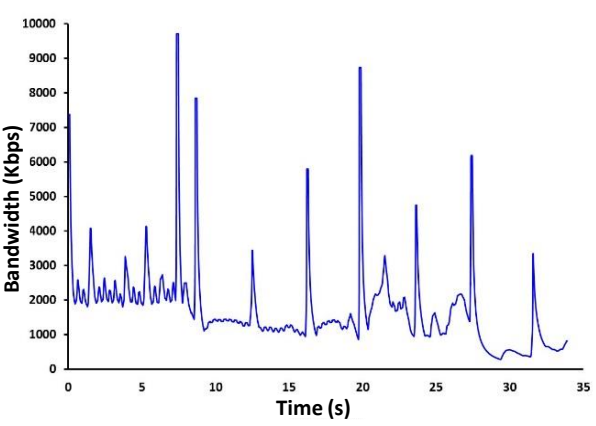

Fig. 9. Bandwidth wireless base line.

\subsection{Study of the Internal Network Parameters and the Transmission Effects}

In this section we study the effect on the multimedia traffic. Concretely, we study, the video quality perceived when we modify internal parameters of the network configuration and protocols such as the transmission rate of WAN links, and when we decrease the available bandwidth by increasing other traffic type.

1) Effects of Speed Transmission in WAN Links - Point to Point

In this first case study we measure the video reception, in both wired and wireless environments, by varying the transmission speed of the WAN links. The values of the bandwidth selected for the study are 2 Mbps, 4 Mbps and 5.3 Mbps. The design proposed for the studio is observed in Figure 10.

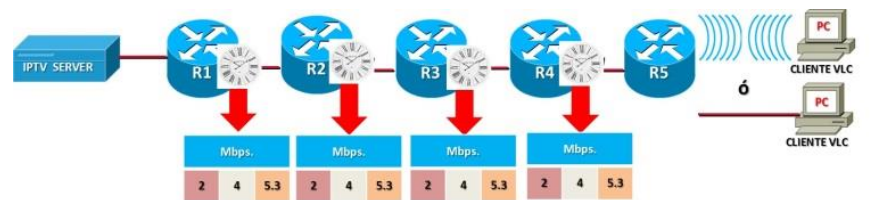

Fig. 10. Transmission from the IPTV server to the VLC Client using different WAN links.

After the analysis of the network parameters captured during video delivery through the WAN links at different speeds, we obtained the following results. Regarding the delay, we observe that at a transmission speed of 2 Mbps in the WAN links, the average value obtained is $6.2 \mathrm{~ms}$. However, for $4 \mathrm{Mbps}$ and $5.3 \mathrm{Mbps}$ the average was $7.36 \mathrm{~ms}$, in both wired and wireless (it is shown in figure 11).

Concerning the jitter, we obtain different values for wired than wireless. For a transmission rate of 2 Mbps in WAN links, the obtained average results are superior when the links are 5.3 Mbps and $4 \mathrm{Mbps}$, as it can be observed in figure 12.

To determine the average percentage of lost packets, we performed the following calculations. First, we subtracted the sequence of packets between the last and the first visible package. Then, the number of packets is obtained, which in this case is 5092. Later, we estimated the difference between the sequence number of packets minus the number of packets (this is the number of lost packets). Finally, we divide the number of lost packets by the packet sequence, thus obtaining the percentage of lost packets. 


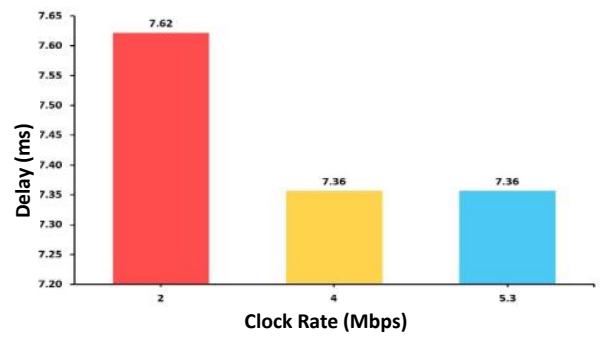

Fig. 11. Average delay at different transmission speeds in the WAN links.

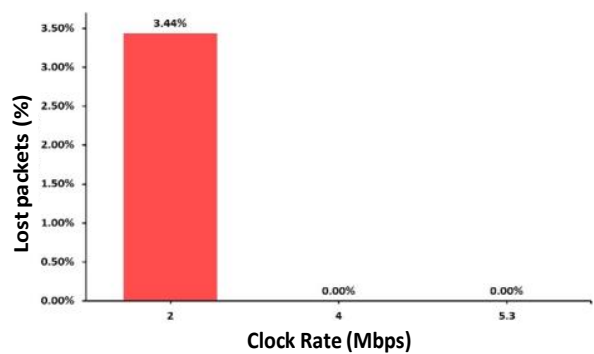

Fig. 13. Average lost packets for different transmission speeds of the WAN links.

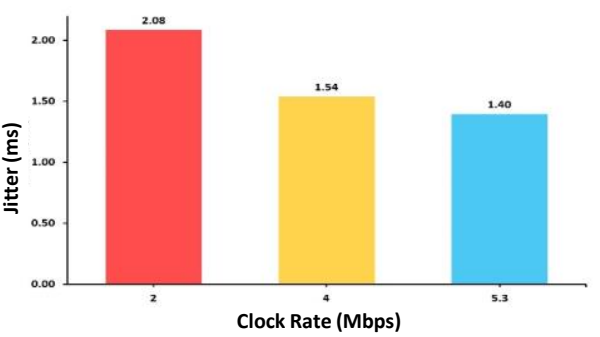

Fig. 12. Average jitter at different transmission speeds in the WAN links.

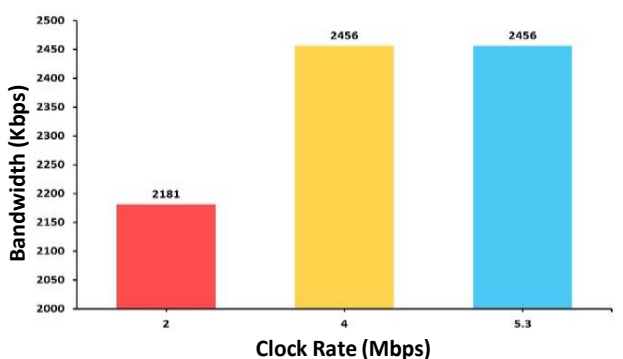

Fig. 14. Average bandwidth for different transmission speeds of the WAN links.

We can see in figure 13 that with a transmission rate of 2 Mbps at WAN links, the average percentage of lost packets is $3.44 \%$ for wired environments, and $3.48 \%$ for wireless. For a transmission speed of 4 Mbps and 5.3 Mbps in WAN links, we obtained an overall average of $0 \%$. This means that there are no packet losses for both cases in the reception. Given that there is a percentage of loss in estimating the transmission speed of 2 Mbps WAN link, we evaluate in this particular case which is the number of packets lost during the delivery and thus make a proper balance.

In figure 14, it is shown the average bandwidth of the video streams obtained when we vary the transmission speed in WAN links at $2 \mathrm{Mbps}, 4 \mathrm{Mbps}$ and $5.3 \mathrm{Mbps}$. For a WAN link with 2 Mbps, there was an average value of 2181 Kbps. For 4 Mbps and 5.3 Mbps we observed an average value of $2456 \mathrm{Kbps}$.

\section{Result Analysis}

In this section, we analyze the results we have obtained in the previous section. We carry out an analysis of the statistical variance to compare the effect of the studied parameters in relation to the base line in order to test the null hypothesis $\mathrm{HO}$ defined in each case. The null hypothesis $\mathrm{H} 0$ is defined as follows. $\mathrm{H} 0$ is met when there is no statistically significant difference among the parameters: average latency, jitter, packet loss or bandwidth of each measure compared to those in the baseline. A value of $\alpha=0.01$ is established to carry out the calculation. We focus our study only on the analysis of the internal parameters in multimedia transmissions, because the results of the effect of external ones have not been conclusive. We compare the results obtained in these cases with the baseline. 
In this case, we change the transmission speed of the WAN links in the wired LAN and study the QoS parameters. The statistical calculations summary of the delay is shown in Table 1. If we establish the transmission speed of the WAN links to 2Mbps the average delay value is $7.62 \mathrm{~ms}$. Statistically, it shows a significant difference, but from the point of view of QoS, it may be considered negligible or insignificant. Likewise, we cannot exclude the results with the other experimental conditions: 4 Mbps and 5.3 Mbps. Therefore, it indicates that with those values an increment of the link bandwidth does not affect the latency behavior respect to the one obtained in the baseline. In Table 2, we show the summary of the statistical calculations for the jitter. The average jitter value is $2.08 \mathrm{~ms}$. when we establish the transmission speed of the WAN link to $2 \mathrm{Mbps}$ and $1.54 \mathrm{~ms}$ when we establish it to $4 \mathrm{Mbps}$. The jitter obtained for $2 \mathrm{Mbps}$ and $4 \mathrm{Mbps}$ is out of the confidence interval range established in the baseline measure. Therefore, we found in this experiment, with a $99.9 \%$ of guarantee, that when the transmission rate of the WAN links is $2 \mathrm{Mbps}$ or $4 \mathrm{Mbps}$, there is a significant increase of the jitter. Likewise, the jitter when there is a transmission speed of $5.3 \mathrm{Mbps}$ in the WAN links is $1.40 \mathrm{~ms}$. There is a significant difference from the statistical point of view. However, we consider it negligible from the viewpoint of the QoS. The summary of the statistical calculations for packet loss in shown in Table 3. The average value of packet loss is $3.46 \%$ when the transmission speed of the WAN links is established to $2 \mathrm{Mbps}$. That value is out of the confidence interval range established in the baseline measure. Therefore, we found in this experimental condition, with a $99.9 \%$ of guarantee, that when the transmission speed of the WAN links is $2 \mathrm{Mbps}$, there is a significant increase in the number of packet loss. We cannot exclude the difference of the other experiments: $4 \mathrm{Mbps}$ and 5.3 Mbps. They indicate that an increment of the bandwidth in the WAN link, respect to those initially shown in the baseline, does not affect to the packet loss. To sum up, in this first case, we can see that the transmission is affected when the transmission speed of the WAN links is reduced to $2 \mathrm{Mbps}$. The most affected parameter is the packet loss. Jitter is affected in a lesser grade. Delay is not affected.

Table 1. Study of the delay when comparing the baseline with different transmission speeds of the WAN Links.

\begin{tabular}{|c|c|c|c|c|c|}
\hline \multirow{3}{*}{$\begin{array}{l}\text { Transmission } \\
\text { speed of WAN } \\
\text { links (Mbps.) }\end{array}$} & \multicolumn{2}{|c|}{ Network parameters } & \multicolumn{3}{|c|}{ Statistical parameters } \\
\hline & \multirow{2}{*}{$\begin{array}{c}\text { Number of } \\
\text { packets (bytes) }\end{array}$} & \multirow{2}{*}{$\begin{array}{c}\text { Delay } \\
\text { (ms) }\end{array}$} & \multirow{2}{*}{$\begin{array}{c}\text { Standard } \\
\text { Deviation }(\mathrm{ms})\end{array}$} & \multicolumn{2}{|c|}{ Subhead } \\
\hline & & & & Min. (ms) & $\operatorname{Max} .(m s)$ \\
\hline Base Line & 5092 & 7.36 & 7.10 & 7.10 & 7.61 \\
\hline 2 & 4917 & 7.62 & 5.76 & 7.41 & 7.83 \\
\hline 4 & 5092 & 7.36 & 6.86 & 7.11 & 7.60 \\
\hline 5.3 & 5092 & 7.36 & 7.03 & 7.10 & 7.61 \\
\hline
\end{tabular}

Table 2. Study of the jitter when comparing the baseline with different transmission speeds of the WAN Links.

\begin{tabular}{|c|c|c|c|c|c|}
\hline \multirow{2}{*}{$\begin{array}{c}\text { Transmission } \\
\text { speed of WAN } \\
\text { links (Mbps.) }\end{array}$} & \multicolumn{2}{|c|}{$\begin{array}{c}\text { Network parameters } \\
\text { Number of } \\
\text { packets } \text { (bytes) }\end{array}$} & $\begin{array}{c}\text { Jitter } \\
(\boldsymbol{m} \text { s) }\end{array}$ & $\begin{array}{c}\text { Standard } \\
\text { Deviation }(\boldsymbol{m s})\end{array}$ & \multicolumn{2}{c|}{ Min. (ms) } & Max.(ms) \\
\hline Base Line & 5092 & 1.37 & 0.70 & 1.34 & 1.39 \\
\hline 2 & 4917 & 2.08 & 1.55 & 2.03 & 2.14 \\
\hline 4 & 5092 & 1.54 & 0.83 & 1.51 & 1.57 \\
\hline 5.3 & 5092 & 1.40 & 0.75 & 1.37 & 1.42 \\
\hline
\end{tabular}


Table 3. Study of the packet loss when comparing the baseline with different transmission speeds of the WAN Links.

\begin{tabular}{|c|c|c|c|c|c|}
\hline \multirow{3}{*}{$\begin{array}{l}\text { Transmission } \\
\text { speed of WAN } \\
\text { links (Mbps.) }\end{array}$} & \multicolumn{2}{|c|}{ Network parameters } & \multicolumn{3}{|c|}{ Statistical parameters } \\
\hline & \multirow{2}{*}{$\begin{array}{c}\text { Number of } \\
\text { packets (bytes) }\end{array}$} & \multirow{2}{*}{$\begin{array}{c}\text { Packet } \\
\text { loss (ms) }\end{array}$} & \multirow{2}{*}{$\begin{array}{c}\text { Standard } \\
\text { Deviation }(\mathrm{ms})\end{array}$} & \multicolumn{2}{|c|}{ Subhead } \\
\hline & & & & Min. (ms) & $\operatorname{Max} .(\mathrm{ms})$ \\
\hline Base Line & 5092 & 0.00 & 0.00 & 0.00 & 0.00 \\
\hline 2 & 4917 & 3.46 & 18.27 & 2.79 & 4.13 \\
\hline 4 & 5092 & 0.00 & 0.00 & 0.00 & 0.00 \\
\hline 5.3 & 5092 & 0.00 & 0.00 & 0.00 & 0.00 \\
\hline
\end{tabular}

\section{Conclusion}

We have implemented a test bench to observe both the network traffic QoS parameters variations of the received video and the impact in the image quality due to the network parameters variations. We have designed, assembled and configured a WAN network for multimedia traffic transmission. To carry out the test we have used routers, switches, PCs and an access point. We have obtained measurements from the test bench and we have analyzed the significant differences in order to evaluate the QoS parameters basing it on a variance statistical analysis of the different study cases.

We have noticed a significant packet loss when the WAN point-to-point links transmission speed values decrease to near the video transmission average bandwidth ( $2 \mathrm{Mbps}$ ). Some significant changes (but less) are also noticed in the jitter. Delay parameter is not significantly affected.

The study results show that service operators should have both video and QoS tests, in order to have a data transmission statistical database (video, voice, etc.). As far as multimedia communication services demand tends to grow every day, it is necessary to deliver reliability and confidence to customers in order to offer them a good QoS. Therefore, that testbech should be able to reproduce the conditions in both wired and wireless environments.

In future works we intend to use the testbench defined to test the received video quality using different types of queues, video streaming applications quality, and different video codecs performance and quality after the transmission. Moreover, due to the proliferation of the video streaming applications for Content Delivery Networks (CDNs), Peer-to-Peer (P2P) networks, Multimedia Cloud Computing, etc., we will use the implemented testbench in new streaming applications. In addition, it will allow us to test on any device (laptops, phones, etc.) the performance and quality of different video and audio codecs after transmission [17].

\section{References}

[1] J. Lloret, M. Garcia, M. Atenas, A. Canovas, A QoE management system to improve the IPTV network, International Journal of Communication Systems 24 (1), 118-138. 2011

[2] M. Garcia, A. Canovas, M. Edo, J. Lloret, A. QoE management system for ubiquitous IPTV devices, The Third International Conference on Mobile Ubiquitous Computing, Systems, Services and Technologies. UBICOMM 2009. October 11-16, 2009, Sliema, Malta. 
[3]A. Álvarez, L. Pozueco, S. Cabrero, X. García Pañeda, R. García, D. Melendi, G. Díaz Orueta, Adaptive Streaming: A subjective catalog to assess the performance of objective QoE metrics, Network Protocols and Algorithms, Vol 6, No 2 (2014). Pp. 123-136

[4] J. M. Jimenez, O. Romero, A. Rego, A. Dilendra, J. Lloret, Study of Multimedia Delivery over Software Defined Networks, Network Protocols and Algorithms, Vol 7, No 4 (2015), Pp. 37-62

[5]Recomendation UIT/T G.1010: End-user multimedia QoS categories (2001). At: https://www.itu.int/rec/T-REC-G.1010-200111-I/en

[6] Recomendation UIT- R M.1079-2: Performance and quality of service requirements for International Mobile Telecommunications-2000 (IMT-2000) access networks. At: https://www.itu.int/dms_pubrec/itu-r/rec/m/R-REC-M.1079-2-200306-I!!PDF-E.pdf

[7] M. Garcia, J. Lloret, M. Edo, R. Lacuesta, IPTV distribution network access system using WiMAX and WLAN technologies, Proceedings of the 4th edition of the UPGRADE-CN workshop on Use of P2P GRID and agents for the development of content networks. Munich, Germany, June 11 - 13, 2009

[8] M. Atenas, S. Sendra, M. Garcia, J. Lloret, IPTV Performance in IEEE 802.11 n WLANs, IEEE GLOBECOM Workshops 2010, Miami (USA); December 2010. p. 929-33.

[9] Cisco Systems Inc. Cisco's Visual Networking Index Forecast Projects Nearly Half the World's Population Will Be Connected to the Internet by 2017, http://newsroom.cisco.com/press-release-content?articleId=1197391

[10] X. Lu, S. Tao, M. El Zarki, R. Guérin, Quality based Adaptive Video Over the Internet. Dept of ESE, University of Pennsylvania, Philadelphia, PA 19104 (2003)

[11] T. K. Lee, T. Y. Chai, L. H. Ngoh, X. Shao, J. C. M. Teo, \& L. Zhou, An IMS-based testbed for real-time services integration and orchestration. IEEE Asia-Pacific Services Computing Conference, (APSCC 2009). Singapore, December 7-11 2009,

[12] M. Mu, A. Mauthe, J. Casson, G. Tyson, \& F. Garcia, LA1 TestBed: evaluation testbed to assess the impact of network impairments on video quality. 5th International Conference on Testbeds and Research Infrastructures for the Development of Networks \& Communities and Workshops, (TridentCom 2009). April 6 8, 2009, Washington DC

[13] A. Kuwadekar, K. Al-Begain, User Centric Quality of Experience Testing for Video on Demand over IMS. First International Conference on Computational Intelligence, Communication Systems and Networks (CICSYN'09), Indore, India, 23-25 July 2009

[14] Downline Guide, Big Buck Bunny, Sunflower version, http://bbb3d.renderfarming.net/download.html

[15] FFmpeg, At: http://www.ffmpeg.org/about.html

[16] G. J. Sullivan, \& T. Wiegand, Video Compression - From Concepts to the H.264/AVC

Standard. Proceedings of the IEEE, Vol. 93, No. 1, (2005)

[17] A. J. Estepa, R. Estepa, J. Vozmediano, P. Carrillo, Dynamic VoIP codec selection on smartphones, Network Protocols and Algorithms, Vol 6, No 2 (2014). Pp. 22-37 